\title{
Entropy and Irreversibility in Classical and Quantum Mechanics
}

\author{
V. A. Antonov ${ }^{1}$, Boris P. Kondratyev ${ }^{1,2}$ \\ ${ }^{1}$ Main (Pulkovo) Astronomical Observatory, Saint Petersburg, Russia \\ ${ }^{2}$ Udmurt State University, Izhevsk, Russia \\ E-mail: kond@uni.udm.ru \\ Received March 3, 2011; revised April 24, 2011; accepted May 12, 2011
}

\begin{abstract}
Review of the irreversibility problem in modern physics with new researches is given. Some characteristics of the Markov chains are specified and the important property of monotonicity of a probability is formulated. Using one thin inequality, the behavior of relative entropy in the classical case is considered. Further we pass to studying of the irreversibility phenomena in quantum problems. By new method is received the Lindblad's equation and its physical essence is explained. Deep analogy between the classical Markov processes and development described by the Lindblad's equation is conducted. Using method of comparison of the Lindblad's equation with the linear Langevin equation we receive a system of differential equations, which are more general, than the Caldeira-Leggett equation. Here we consider quantum systems without inverse influence on a surrounding background with high temperature. Quantum diffusion of a single particle is considered and possible ways of the permission of the Schrödinger's cat paradox and the role of an external world for the phenomena with quantum irreversibility are discussed. In spite of previous opinion we conclude that in the equilibrium environment is not necessary to postulate the processes with collapses of wave functions. Besides, we draw attention to the fact that the Heisenberg's uncertainty relation does not always mean the restriction is usually the product of the average values of commuting variables. At last, some prospects in the problem of quantum irreversibility are discussed.
\end{abstract}

Keywords: Markov Chains, Irreversibility in Classical and Quantum Mechanics, Lindblad Equation, Caldeira-Leggett Equation, Quantum Diffusion, Schrödinger's Cat Paradox, Heisenberg's Uncertainty Relation, Collapse of Wave Function, Effect of Sokolov

\section{Introduction}

The concept of irreversible changes in physics is manifold. First of all, it means a tendency to thermodynamic balance, but the irreversibility problem isn't settled by it. In particular, in modern physics the important role plays an irreversible mixing (or collapses of wave functions) of quantum measurements results. Quantitative measure of an irreversibility is the entropy and connected with it characteristics. Some questions about dissipative quantum systems were considered in [1]. From our point of view, in this sort of questions many doubtful interpretations have collected; therefore in the given work we discuss debatable moments.

In Section 2 some properties of the Markov chains are specified and important property of the probability be- havior to monotony is formulated. Using one thin inequality, in Section 3 behavior of the relative entropy in classical case is considered. Further we pass to studying of the irreversibility phenomena in quantum problems. In Section 4 by new method we receive the Lindblad's equation for density function, and for the first time a physical substantiation to this equation is given. In the same place a deep analogy between the classical Markov processes and development described by the Lindblad equation is given. In Section 5 using the method of comparison of the Lindblad equation with the classical linear Langevin equation we receive a system of differential equations, which are more general, than the Caldeira-Leggett equation. Further we consider quantum systems without return influence on a surrounding background. In Section 7 the quantum diffusion of a single 
particle is considered. Possible ways of the permission of the Schrödinger's cat paradox are discussed in Section 8. Then the role of an external world for the phenomena with quantum irreversibility is considered. In Section 10 we conclude that in the equilibrium environment is not necessary to postulate the processes with collapse of the wave functions. In Section 11 ones draw attention to the fact that the Heisenberg's uncertainty relation does not always mean the restriction is usually the product of the average values of commuting variables. At last, in Section 12 we discuss some prospects in the problem of quantum irreversibility.

\section{Some New Remarks on the Markov Processes}

In the formulation of rules of asymptotic behavior of the Markov chains not to do without the condition, intuitively enough transparent, which we for brevity name here a connectivity condition. Its essence-in the impossibility assumption to break set of conditions into two nonempty subsets I and II so that transitions from I in II were absent, that is that all corresponding transitive probabilities $p_{i j}$ are vanished. Subject to the condition of connection are only two possible types of asymptotic behavior: 1) or for any initial distribution is obtained in the limit - either directly or in sense of the Fejer arithmetic mean - the same final steady - state distribution (reservation is needed about the arithmetic mean for the sake of a few exceptional cases, a periodic or almost periodic behavior in the limit $\left.P_{i}(t)\right) ; 2$ ) or also for any initial distribution all $P_{i}(t)$ tend to zero, the scattering takes place at infinity.

At finite number of states there is, of course, only the first opportunity.

The property of monotony formulated above can't be found in known managements on the Markov processes, see, for example, [2-6]. It can be considered as quantitative expression of an asymptotics in the case 1).

At transition to continuous time a basis of the Markov theory processes remains, but possibility of aforementioned recurrence disappears; but there are complications of type of distinction "simply Markov" and "strict Markov" properties, occurrences of singularities for final time etc., that, however, has not enough relation to the discussed theme. At continuity of transitions in space of conditions diffusive process (for example, in one-dimensional case) is received

$$
\frac{\partial f}{\partial t}=-\frac{\partial}{\partial x}(a f)+\frac{1}{2} \frac{\partial^{2}}{\partial x^{2}}(D f),
$$

where $a(x)$ and $D(x)$-factors accordingly regular displacement and diffusion. For the process (1) monot- ony of the relative entropy (see lower) is easily proved directly. For the Markov chains, symmetric or led by that, the proof (1) is in essence given in [5].

In symmetric case at final $n$ the equiprobability $P_{1}=P_{2}=\cdots=P_{n}=\frac{1}{n}$ (the condition of connectivity entered above here is supposed) is limit, and at infinite $n$ unlimited dispersion turns out $P_{i} \rightarrow 0$. The symmetric variant of the diffusive process (1) is

$$
\frac{\partial f}{\partial t}=\frac{1}{2} \frac{\partial}{\partial x}\left(D(x) \frac{\partial f}{\partial x}\right)
$$

and besides there is the limiting uniform distribution, if the boundaries are entered from both parties obviously (with a condition not disappearances of the particles $\frac{\partial f}{\partial x}=0$ on them) or indirectly, through vanishing $D(x)$ on final distance. For absence of the boundaries there is dispersion on infinity.

\section{The Relative Entropy in Classical Problems}

Let's begin with random processes without feedback for which typical example are the classical Markov chains, having numerous applications in physics. The basic equation of such chains with final number $N$ of the conditions is $[2,3]$

$$
P_{j}^{(m)}=\sum_{i=1}^{N} P_{i}^{(m-1)} p_{i j},
$$

$m$-number of discrete step, indexes $i$ and $j$ number the conditions, $P_{i}$ - probability of stay of system in the condition $i$, and $p_{i j}$-the conditional probability of transition $i \rightarrow j$. The obvious conditions should be satisfied

$$
\begin{gathered}
P_{i}>0, \quad \sum_{i=1}^{N} P_{i}=1 \quad(\text { under any } m) ; \\
p_{i j}>0, \quad \sum_{j=1}^{N} p_{i j}=1 .
\end{gathered}
$$

For the system (3) should be the stationary solution $Q_{i}$

$$
Q_{j}=\sum_{j=1}^{N} Q_{i} p_{i j}
$$

with the same restrictions (5). On the each step it is possible to form the function of $N$ real variables $\lambda_{1} \cdots \lambda_{N}$

$$
F_{m}\left(\lambda_{1}, \lambda_{2}, \cdots, \lambda_{N}\right)=-\sum_{i=1}^{N} \lambda_{i} P_{i}^{(m)}+\sum_{i=1}^{N} Q_{i} e^{\lambda_{i}} .
$$

The minimum of this function is in the usual way at

$$
-P_{i}^{(m)}+Q_{i} e^{\lambda_{i}}=0, \quad \lambda_{i}=\ln \frac{P_{i}^{(m)}}{Q_{i}} .
$$


It is equal

$$
\min F_{m}=-\sum_{i=1}^{N} P_{i}^{(m)} \ln \frac{P_{i}^{(m)}}{Q_{i}}+\sum_{i=1}^{N} P_{i}^{(m)}=1+\widetilde{S}_{m},
$$

where $\tilde{S}_{m}$ is the relative entropy

$$
\widetilde{S}_{m}=-\sum_{i=1}^{N} P_{i}^{(m)} \ln \frac{P_{i}^{(m)}}{Q_{i}} .
$$

Let's prove that the sequence $\widetilde{S}_{m}$ monotonously increases. From (3) and (7) follows

$$
F_{m}=-\sum_{i=1}^{N} \sum_{j=1}^{N} \lambda_{i} P_{j}^{(m-1)} p_{j i}+\sum_{i=1}^{N} Q_{i} \mathrm{e}^{\lambda_{i}} .
$$

Enter the indications

$$
\lambda_{j}^{\prime}=\sum_{i=1}^{N} \lambda_{i} p_{j i}
$$

then

$$
F_{m}=-\sum_{j=1}^{N} \lambda_{j}^{\prime} P_{j}^{(m-1)}+\sum_{j=1}^{N} Q_{j} \mathrm{e}^{\lambda_{j}} .
$$

As the exponential belongs to number of the convex functions, taking into account (5) from the Jensen's inequality follows [7]

$$
\mathrm{e}^{\lambda_{j}^{\prime}} \leq \sum_{i=1}^{\infty} p_{i j} \mathrm{e}^{\lambda_{j}}
$$

From the equality (7) and the relation (8) we obtain

$$
1+\widetilde{S}_{m-1} \leq-\sum_{i=1}^{N} \lambda_{i}^{\prime} P_{i}^{(m-1)}+\sum_{i=1}^{N} Q_{i} \mathrm{e}^{\lambda_{i}^{\prime}}
$$

where the last member is estimated by means of (12) and (6):

$$
\sum_{j=1}^{N} Q_{i} \mathrm{e}^{\lambda_{j}^{\prime}} \leq \sum_{j=1}^{N} \sum_{i=1}^{N} p_{i j} Q_{j} \mathrm{e}^{\lambda_{i}}=\sum_{i=1}^{N} Q_{i} \mathrm{e}^{\lambda_{i}}
$$

So, after comparison with (11), from (13) we have

$$
1+\widetilde{S}_{m-1} \leq F_{m}\left(\lambda_{1}^{\prime}, \cdots, \lambda_{N}^{\prime}\right)
$$

where, in particular, the parameters $\lambda^{\prime}$ can be chosen so that they minimized the right part. Then

$$
\widetilde{S}_{m-1} \leq \widetilde{S}_{m}
$$

as was to be shown.

Exact equality in (14) is reached in the stationary state $P_{i}=Q_{i}$. (By the way $\widetilde{S}=0$, thus in general case $\widetilde{S}<0$ ). Limiting relation

$$
\lim _{m \rightarrow \infty} P_{i}^{(m)}=Q_{i}
$$

can be broken only in special cases of disintegration of set conditions on subsets, the transitions between which are absent.
Let's underline that the monotonous change of the relative entropy doesn't contradict possible sometimes to reduction of the usual (absolute) entropy

$$
S=-\sum_{i=1}^{N} P_{i} \ln P_{i}
$$

The typical example is the diffusion of relatively heavy molecules in gas in the presence of cold wall. The molecules are eventually collected on this wall and the final distribution $Q$ takes a form of the Dirac $\delta$ function-this is clear example of reducing of the entropy. Physically, it is obvious that the case is characterized by increase in the total entropy in the transfer of heat from the gas to the cold wall. Similarly, a massive test body (for example, globular stellar cluster) moving in the galaxy is experiencing dynamic friction [8] and eventually up to some fluctuations, gradually settles to its center.

The relative entropy coincides with the absolute entropy at the independence $Q_{i}$ of an index, i.e. when $Q_{i}=\frac{1}{N}$, for which enough (but it is not necessary) that there was symmetry of stochastic process: $p_{i j}=p_{j i}$.

\section{Irreversibility in the Quantum Case}

The irreversible processes in quantum mechanics can be studied either by setting a probability distribution for parameters of the wave function (in the case of pure states), or in terms of the density function (the states are mixed). However, in both cases, the irreversibility manifests itself only in the interaction of a quantum system with exterior reservoir or bath. The wave function of a dedicated system, in principle, refused to serve when you can not ignore its inverse effect on the surrounding background-i.e. when takes place a link, or entanglement of states of the system and the thermostat [9]. Regarding the density function believe that it is sufficient to completely describe a single system (as indicated below, also gave rise to some doubts), but it certainly is not enough when there are many individual systems in the general context [10].

Let's start with the density function $\rho$. Lindblad [11] derived the most general law of evolution $\rho(t)$, compatible with conservation of normalization, positive definiteness of the function, the lack of aftereffects (and some of the requirements of continuity and uniqueness). Should be

$$
\frac{\partial \rho}{\partial t}=-\frac{i}{\hbar}[H, \rho]+\sum_{v=1}^{n}\left(-L_{v}^{+} L_{v} \rho+2 L_{v} \rho L_{v}^{+}-\rho L_{v}^{+} L_{v}\right),
$$

where $L_{j}$-any operators, $H$-the Hermit operator. (That circumstance that in (3) time was discrete, doesn't 
play an essential role).

But Lindblad hasn't given a physical substantiation to the Equation (16). We eliminate this omission and will make this as follows.

Let the chosen object interacts from time to time during a short interval $0<t<\delta t$ with external field making a part of the background and before not dependent on given object. Actually, it is the Boltzmann scheme, which though was studied earlier at quantum level, but only at private assumptions [12,13]. To derive Equation (16), we apply the general scheme. At the moment $t=0$ there is a need to use the new density matrix $\rho$ as a direct product of the density matrix $\bar{\rho}$ and the density matrix of external field. For simplicity, assume that $\overline{\overline{\rho_{1}}}$ has dimension $2 \times 2$. In diagonal form, hence,

$$
\overline{\overline{\rho_{1}}}(0)=\left(\begin{array}{cc}
\xi_{1} & 0 \\
0 & \xi_{2}
\end{array}\right), \quad \xi_{1}>0, \quad \xi_{2}>0, \quad \xi_{1}+\xi_{2}=1
$$

Accordingly, $\underline{\underline{\rho}}(0)$ has a usual form

$$
\begin{aligned}
& \underline{\rho}=(0)=\left(\begin{array}{cc}
\xi_{1} \rho & 0 \\
0 & \xi_{2} \rho
\end{array}\right) . \\
& \underline{\underline{\rho}}(\delta t)=\bar{\rho}(0) e^{-\frac{i\langle H\rangle \delta t}{\hbar}} \\
& \langle H\rangle=\xi_{1} H_{11}+\xi_{2} H_{22}, \\
& L_{1}=\sqrt{\xi_{1} \xi_{2}}\left(H_{11}-H_{22}\right), \\
& L_{2}=\sqrt{\xi_{1}} H_{21}, \\
& L_{3}=\sqrt{\xi_{2}} H_{12} .
\end{aligned}
$$$$
\underline{\underline{\rho}}(\delta t)=\bar{\rho}(0) e^{-\frac{i\langle H\rangle \delta t}{\hbar}} e^{\frac{i\langle H\rangle \delta t}{\hbar}}+\frac{(\delta t)^{2}}{2 \hbar^{2}} \sum_{k=1}^{3}\left(L_{k}^{+} L_{k} \bar{\rho}(0)+\bar{\rho}(0) L_{k}^{+} L_{k}-2 L_{k} \bar{\rho}(0) L_{k}^{+}\right)
$$

where

Let us imagine now that the whole process of evolution has described the same type of interactions. The background for this is stationary, but, generally speaking, with the internal nonequilibrium (anisotropy of the temperature, etc.). It is easy to see that the return of (21) to a continuous smoothed time results with the same accuracy $(\delta t)^{2}$ to the Lindblad Equation (16). In larger dimension
For the Hamiltonian operating all system on interval $(0, \delta t)$, we take as much as possible general form

$$
H=\left(\begin{array}{ll}
H_{11} & H_{12} \\
H_{21} & H_{22}
\end{array}\right), \quad\left(H_{21}=H_{12}^{+}\right)
$$

Then

$$
\underline{\underline{\rho}}(\delta t)=\mathrm{e}^{-\frac{i H \delta t}{\hbar}} \underline{\underline{\rho}}(0) \mathrm{e}^{\frac{i H \delta t}{\hbar}} .
$$

Expansion on powers of $\delta t$ do up to second order terms

$$
\begin{aligned}
\underline{\rho}(\delta t)= & \underline{\rho}(0)+\frac{i \delta t}{\hbar}(\underline{\underline{\rho}}(0) H-H \underline{\underline{\rho}}(0)) \\
& +\frac{t^{2}}{2 \hbar^{2}}\left(2 H \underline{\underline{\rho}}(0) H-H^{2} \underline{\underline{\rho}}(0)-\underline{\rho}=(0) H^{2}\right)+\cdots
\end{aligned}
$$

After interaction stopping, by the general rule it is necessary to take "trace" $\underline{\underline{\rho}}(\delta t)$ and to return to former dimension for $\underline{\underline{\rho}}(\delta t)$. As a result, with the same accuracy we obtain $m \times m$ of the matrix $\overline{\overline{\rho_{1}}}$ instead of (22) appears broader set of the operators

$$
\begin{aligned}
& \sqrt{\xi_{k} \xi_{i}}\left(H_{i i}-H_{k k}\right), \quad \sqrt{\xi_{i}} H_{k}, \\
& \sqrt{\xi_{k}} H_{i}, \quad(1 \leq i<k \leq m) .
\end{aligned}
$$

The two-level system concerns to number of the simplest examples. It is convenient to consider $\rho$ as combination from a scalar part and the Pauli matrix accepted further for the coordinate unit vectors. Then $\rho$ it is possible to write down as $\left(\rho_{0}, \boldsymbol{\rho}\right)$, similarly in the vector form undertake $H$ and $L$ (their scalar parts don't give the contribution). Obviously, $\rho_{0}$ remains, and $\rho$ evolves according to (15) under the law

$$
\frac{\partial \rho}{\partial t}=\frac{1}{\hbar}(\boldsymbol{H} \times \boldsymbol{\rho})+2 i \rho_{0}\left(\boldsymbol{\alpha} \times \boldsymbol{\alpha}^{+}\right)+\boldsymbol{\alpha}^{+} \times(\boldsymbol{\alpha} \times \boldsymbol{\rho})+\boldsymbol{\alpha} \times\left(\boldsymbol{\alpha}^{+} \times \boldsymbol{\rho}\right) .
$$

In particular, if to consider $|\rho|$ and $\|\boldsymbol{\alpha}\|$ as small one order, and $|\boldsymbol{H}|=0$, in the right part (16) the second member prevails, and it can be oriented, for example,

$$
S\{\boldsymbol{\rho}\}=-S p(\boldsymbol{\rho} \ln \rho)=-\left(\rho_{0}+|\rho|\right) \ln \left(\rho_{0}+|\boldsymbol{\rho}|\right)-\left(\rho_{0}-|\boldsymbol{\rho}|\right) \ln \left(\rho_{0}-|\boldsymbol{\rho}|\right)
$$


also can change in any party.

Pulè [14] gives the evolution equation, very similar to (24) and essentially equivalent to it, as he also highlighted and a systematic component, similar to the first two terms on the right side (24), and "damped" component, similar the last two terms we have in (24). This correspon-dence is observed, despite the private nature of the model in [14].

Thus, there are foundations to consider (16) in general analog of the classical stochastic equation of evolution (1).

The analogy between the quantum process of type (21) and the classical Markov chains is represented to us rather deep. First of all, for bounded $n$ always there is a decision of the Equation (21). Really, at any initial positively defined $\rho(0)$ the arithmetic mean

$$
g(T)=\frac{1}{T} \int_{0}^{T} \rho(t) \mathrm{d} t
$$

preserves properties of positive definiteness and the unit trace, and consequently, the uniformly regular bounded elements. Moreover, if denote by A superoperator on the right side (21), that

$$
A\{g\}=\frac{1}{T} \int_{0}^{T} \frac{\mathrm{d} \rho}{\mathrm{d} t} \mathrm{~d} t=\frac{\rho(T)-\rho(0)}{T}
$$

tends to zero, and thus, in the limit of at least some increasing sequence of values of $T$ is obtained the steadystate solution (21).

Use of the logarithmic function of the matrices in this context is formally inconvenient. Instead of, it can make the quadratic measure of closeness, which varies monotonically. Let's present the stationary matrix $\rho=B$ to diagonal form:

$$
B=\left(\begin{array}{cccc}
b_{1} & 0 & \cdots & 0 \\
0 & b_{2} & \cdots & 0 \\
\cdot & \cdot & \cdot & \cdot \\
0 & 0 & \cdots & b_{n}
\end{array}\right) .
$$

Similarly, the elements of any density matrix $\rho(t)$ we will designate as $\rho_{i k}$. We define the functional

$$
w\{\rho\}=2 \sum_{i, k} \frac{\left|\rho_{i k}\right|^{2}}{b_{i}+b_{k}}-1 .
$$

Term $(-1)$ in (29) is introduced only to the particular case $\rho \equiv B$ to have $w=0$. For $w\{\rho\}$ we will make the variational definition which is simultaneously a generalization on any choice of basis,

$$
w\{\rho\}=\max S p(2 \varphi \rho-\varphi B \varphi)-1,
$$

where the maximum undertakes on any Hermit matrixes $\varphi$ of the same dimension $n \times n$. For check it is varied the functional in the right part (30), replacing $\varphi$ on $\varphi+\delta \varphi$. A corresponding increment is opened as

$$
\begin{aligned}
\Delta & =S p\{2 \delta \varphi \rho-\varphi B \delta \varphi-\delta \varphi B \varphi-\delta \varphi B \delta \varphi\} \\
& =S p\left\{2 \delta \varphi\left(\rho-\frac{B \varphi+\varphi B}{2}\right)\right\}-S p(\delta \varphi B \delta \varphi) .
\end{aligned}
$$

If concretely to choose the matrix $\varphi$ with elements

$$
\varphi_{i k}=\frac{2 \rho_{i k}}{b_{i}+b_{k}},
$$

then the matrix $N=\rho-\frac{B \varphi+\varphi B}{2}$ is zero and in the right part (31) remains the last, negative or zero, member. So, the maximum in (29) is reached at a choice of elements $\varphi$ according to (32). Simple calculation shows that the magnitude of this maximum coincides with (30).

The proof of monotony for $w\{\rho(t)\}$ it is enough to give for the elementary case

$$
\frac{\partial \rho}{\partial t}=-L^{+} L \rho-\rho L^{+} L+2 L \rho L^{+} .
$$

In (33) we uncover dependence $\rho$ and $B$ from a time according to (19), though actually $\frac{\mathrm{d} B}{\mathrm{~d} t}=0$. Dependence $\rho$ on a time can be at first arbitrary if only the admissible set of Hermit $\varphi$ was saved. Concretely, we assume

$$
\frac{\partial \varphi}{\partial t}=L^{+} L \varphi+\varphi L^{+} L-2 L^{+} \rho L .
$$

Then, in particular,

$$
\begin{aligned}
\frac{\mathrm{d}}{\mathrm{d} t} S p(\varphi \rho) & =S p\left(\frac{\mathrm{d} \varphi}{\mathrm{d} t} \rho+\varphi \frac{\mathrm{d} \rho}{\mathrm{d} t}\right) \\
& =S p\left(L^{+} L \varphi \rho-2 L^{+} \varphi \rho-\varphi \rho L^{+} L+2 \varphi L \rho L^{+}\right) \\
& =0,
\end{aligned}
$$

and it is easy to be convinced by a rule of circular permutation. Analogously after differentiation $\varphi B \varphi$ in each term it is done such circular permutation that matrix $B$ has appeared at centre fivefold product. As a result,

$$
\frac{\mathrm{d}}{\mathrm{d} t} \operatorname{Sp}(2 \varphi \rho-\varphi B \varphi)=-2 S p[\varphi, L] B([\varphi, L])^{*} \leq 0 .
$$

Let in instant $t+\delta t$ matrix $B(t+\delta t)$ is selected so that to maximize a track in (36). Then, in the moment, has just been proved, the matrix $\varphi(t)$ associated with the previous equation (36), the value of the track must be more $1+w\{\rho(t+\delta t)\}$. This $\varphi(t)$ will not be, generally speaking, maximized, but when taking the maximum 
the inequality will only intensify:

$$
w\{\rho(t)\} \geq w\{\rho(t+\delta t)\}
$$

as was to be shown.

In deciding whether the standard solution is nondegenerate, i.e., whether it has full rank $n$, again shows the analogy with the Markov chains: degeneration occurs only at infraction of the connectivity condition. We set off such violation, when at suitable unitary transformation, common for all $L_{v}$, it is possible to select two nonempty groups of states $I$ and $I I$ so that in all $L_{v}$ transitions $I \rightarrow I I$ miss.

For the demonstration we will result again the stationary matrix $B$ in diagonal form. If, in former labels, $b_{i} \neq 0(1 \leq i \leq s)$, but $b_{s+1}=\cdots=b_{n}=0(s<n)$, it is computed in $\frac{\mathrm{d} B}{\mathrm{~d} t}$ the diagonal elements with $i>s$, that gives

$$
\frac{\mathrm{d} b_{i}}{\mathrm{~d} t}=\sum_{v=1}^{N} \sum_{k=1}^{s}\left|l_{i k}^{v}\right|^{2}
$$

$\left(l_{i k}^{v}\right.$ - elements of matrix $\left.L_{v}\right)$. The requirement of stationarity is fulfilled, only if all $l_{i k}$ with $i<s, k \leq s$ are vanished, and this means precisely disintegration of set of states on two groups.

At performance of the connectivity condition also easily proved that $\rho(t)$ is aimed to a stationary state at least at terminating $n$. Really, let's will look, when inequality $\frac{\mathrm{d} w}{\mathrm{~d} t} \leq 0$ becomes exact equality. For this purpose at nondegeneracy $B$ commutation $\varphi$ with all $L_{v}$ is necessary. We will realize, for a moment, reduction $\varphi$ to the diagonal form. If among eigenvalues of this matrix are various ones, on a commutation requirement in $L_{v}$ there can not be transitions between states of corresponding groups, i.e. the connectivity condition is broken. Could be considered that the matrix $\varphi$ is proportional the unit matrix, but then $\rho$ and $B$ are proportional each other and if to regard normalization, it is simple $\rho \equiv B$. In any other case the condition $\frac{\mathrm{d} w}{\mathrm{~d} t}<0$ is fulfilled, and from here it is already easy to conclude about an attraction of any state $\rho(t)$ to stationary $B$ (obviously, thus to the only state).

\section{Generalization of the Caldeira-Leggett Equation}

Many authors were engaged in obtaining of a kinematic quantum equations for the case of continuous set of states. Partial survey is given in [1]. However, the premises and conclusions in some cases raise doubts; to trace the cause of contradictions is sometimes difficult. Here we have selected other path: at once we will apply the Lindblad equation in such shape that it could be compared with the linear classical Langeven equation. For this purpose it is possible to take

$$
H=\frac{p^{2}}{2 m}+\frac{m w^{2} q^{2}}{2}, \quad L=v(p-i \eta q)
$$

( $\eta$ is the complex parameter, the magnitude $v$ is real). In [1] author uses similar equation taken from Stenholm, however these arguments are represented to us a little convincing. From a point of sight of symmetry and ease of check our Equation (39) has advantage.

The idea to search Gaussian solutions arises

$$
v \rho\left(x, x^{\prime}\right)=c e^{-\alpha x^{2}-\alpha^{+} x^{\prime 2}+2 \beta x x^{\prime}},
$$

where $\beta$ is real, $c$-also real normalizing coefficient

$$
c=\sqrt{\frac{2(\operatorname{Re} \alpha-\beta)}{\pi}},
$$

and $\operatorname{Re} \alpha>\beta$. Last requirement is necessary and enough for positive definiteness $\rho$ owing to the Meler's formula [15], see also (in less convenient shape) [16]

$$
\begin{gathered}
\frac{1}{\sqrt{1-t^{2}}} \exp \left[\frac{4 x y t-\left(x^{2}+y^{2}\right)\left(1-t^{2}\right)}{2\left(1-t^{2}\right)}\right] \\
=\mathrm{e}^{-\frac{x^{2}+y^{2}}{2}} \sum_{n=0}^{\infty} 2^{-n} H_{n}(x) H_{n}(y) \frac{t^{n}}{n !}
\end{gathered}
$$

Then after substitution (41) in the evolutionary Lindblad equation in our case the system of the ordinary differential equations for $\alpha(t), \beta(t)$ is gained and the normalization should be fulfilled automatically. However more simply to come to the purpose as in [1], composing (linear) equations for the moments of the second order, immediately multiplying the left and the right members (40) on $q^{2}, q p, p q, p^{2}$ and taking matching tracks. So, for the time functions

$$
\begin{aligned}
f(t) & =\left\langle q^{2}\right\rangle=S p q^{2} \rho \\
& =\int x^{2} \rho\left(x, x^{\prime}\right) \mathrm{d} x=\frac{1}{4(\operatorname{Re} \alpha-\beta)}, \\
\varphi(t) & =\left\langle p^{2}\right\rangle=S p p^{2} \rho=-\hbar^{2} \int\left[\frac{\partial^{2}}{\partial x^{2}} \rho\left(x, x^{\prime}\right)\right]_{x^{\prime}=x} \mathrm{~d} x \\
& =\frac{\hbar^{2}\left(|\alpha|^{2}-\beta^{2}\right)}{\operatorname{Re} \alpha-\beta}, \\
\chi(t) & =\frac{1}{2}\langle p q+q p\rangle=i \hbar\left[x \frac{\partial}{\partial x} \rho\left(x, x^{\prime}\right)+\frac{\rho\left(x, x^{\prime}\right)}{2}\right]_{x^{\prime}=x} \\
& =\frac{\hbar \operatorname{Im} \alpha}{2(\operatorname{Re} \alpha-\beta)}
\end{aligned}
$$


we obtain following equations

$$
\begin{aligned}
& \frac{\mathrm{d} f}{\mathrm{~d} t}=\frac{2}{m} \chi+v^{2}\left(2 \hbar^{2}+4 \hbar \operatorname{Re} \eta \cdot f\right), \\
& \frac{\mathrm{d} \varphi}{\mathrm{d} t}=-2 m \omega^{2} \chi+v^{2}\left(2|\eta|^{2} \hbar^{2}+4 \hbar \operatorname{Re} \eta \cdot \varphi\right), \\
& \frac{\mathrm{d} \chi}{\mathrm{d} t}=-m \omega^{2} f+\frac{\varphi}{m}+v^{2}\left(-2 \hbar^{2} \operatorname{Im} \eta+4 \hbar \operatorname{Re} \eta \cdot \chi\right) .
\end{aligned}
$$

The physical treatment of the system (43) is not difficult. The first terms of right members define a characteristic evolution of object. The first terms in brackets give diffusion. By the way, as it is clear and in [1], it is possible to set diffusion preferentially on coordinate if $|\eta|$ it is great. Certainly, the subsequent terms in brackets give a systematic swing of oscillation, if $\operatorname{Re} \eta>0$, and a damping, if $\operatorname{Re} \eta<0$.

For the stationary solution of system (43) it is enough to write out

$$
m^{2} \omega^{2} f+\varphi=-\frac{\hbar\left(m^{2} \omega^{2}+|\eta|^{2}\right)}{2 \operatorname{Re} \eta},
$$

as becomes clear that at $\operatorname{Re} \eta \geq 0$ it is physically unacceptable and even is formally incompatible with any admissible parameters $\alpha$ and $\beta$ in the density function. On the contrary, at $\operatorname{Re} \eta \geq 0$ the system (43) has the unique stationary solution which is simultaneously and limiting one, as at characteristic indexes of matching homogeneous system a real parts are negative.

To build a measure of proximity to stationary state by a common rule - enough bulky operation, but an expression with similar properties is easy enough find immediately. Concretely

$$
\tilde{\omega}=\left(x-\chi_{0}\right)^{2}+\frac{m^{2} \omega^{2}}{2}\left(f-f_{0}\right)^{2}+\frac{\left(\varphi-\varphi_{0}\right)^{2}}{2 m^{2} \omega^{2}},
$$

$\left(f_{0}, \varphi_{0}, \chi_{0}\right.$ - the stationary solution). At $\operatorname{Re} \eta<0$ we have $\frac{\mathrm{d} \tilde{\omega}}{\mathrm{d} t} \leq 0$.

At the arbitrary non-Gaussian shape of a density function the problem on its evolution becomes more complicated. But if to be interested only qualitative asymptotic behavior $\rho\left(x, x^{\prime}, t\right)$, the answer is gained at once: at $\operatorname{Re} \eta<0$ in view of a nondegeneracy of steady state $\rho$ there should be a monotone degree of proximity and consequently the limiting $\rho$ must be always the Gaussian, at least the initial $\rho(0)$ and was not that.

\section{The Systems without Feedback}

The evolutionary equation for the systems subject to casual exterior action was inferred many times [11]. Here we prefer the following common approach. At the regular affecting the evolution of physical system would submit to the law

$$
\rho(t)=\mathrm{e}^{-\frac{i H t}{\hbar}} \rho(0) \mathrm{e}^{\frac{i H t}{\hbar}} .
$$

Let concrete affecting lasts a small interval of time $(0, t)$ then decomposition of a right member (46) on $t$ as to within $O\left(t^{2}\right)$ gives to small parameter

$$
\begin{aligned}
\rho(t)= & \left(1-\frac{i H t}{\hbar}-\frac{H^{2} t^{2}}{2 \hbar^{2}}\right) \rho(0)\left(1+\frac{i H t}{\hbar}-\frac{H^{2} t^{2}}{2 \hbar^{2}}\right)+\cdots \\
= & \rho(0)+\frac{i t}{\hbar}(\rho H-H \rho)-\frac{t^{2}}{2 \hbar^{2}}\left(\rho H^{2}+H^{2} \rho\right) \\
& +\frac{t^{2}}{\hbar^{2}} H \rho H+\cdots
\end{aligned}
$$

If in $H$ there is a randomness, in the right member (47) it is necessary to exchange operators $H, H^{2}$ and $\mathrm{H} \rho \mathrm{H}$ their statistical averages (is marked out by angular brackets). We will compare effect to what would be gained if affecting was spotted at once by average $\langle H\rangle$. The difference as it is easy to see, consists only in terms of the second order on $t$

$$
\rho(t)=e^{-\frac{i\langle H\rangle t}{\hbar}} \rho(0) e^{\frac{i\langle H\rangle t}{\hbar}}+\frac{t^{2}}{2 \hbar^{2}}\left[\rho\langle H\rangle^{2}-\rho\left\langle H^{2}\right\rangle+\langle H\rangle^{2} \rho-\left\langle H^{2}\right\rangle \rho\right]++\frac{t^{2}}{\hbar^{2}}[\langle H \rho H\rangle-\langle H\rangle \rho\langle H\rangle]+\ldots
$$

Let's uncover average in an explicit form through probabilities $\gamma_{j}$ and implementation of matching operators:

$$
\langle H\rangle=\sum_{j=1}^{n} \gamma_{j} H_{j}, \text { etc. }
$$

where $\gamma_{j}>0, \sum_{j=1}^{n} \gamma_{j}=1$. After some rearrangement of terms the expression (48) it is reduced to

$$
\begin{aligned}
\rho(t)= & \mathrm{e}^{-\frac{i\langle H\rangle t}{\hbar}} \rho(0) \mathrm{e}^{-\frac{i\langle H\rangle t}{\hbar}} \\
& +\sum_{i<j} \sum_{i j}\left(2 H_{i j} \rho H_{i j}-H_{i j}^{2} \rho-\rho H_{i j}^{2}\right),
\end{aligned}
$$

with

$$
H_{i j}=\sqrt{\gamma_{i} \gamma_{j}}\left(H_{i}-H_{j}\right) .
$$

Going over to the averaged time, we obtain the Lindblad formula (16) from [11], but with one important 
limitation: the operator $L_{j}$ can only be Hermitian. There is reason to see here an analogy with symmetry of the Markov chain $\left(q_{i j}=q_{j i}\right)$. Anyway, as we now show, the entropy in our example increases monotonically. Indeed, it is easily seen that the first term on the right side (16) has no relation to the change in entropy. If we restrict the discrete set of states, then $\rho$ can be reduced to diagonal form

$$
\rho=\left(\begin{array}{cccc}
\rho_{1} & 0 & \cdots & \cdots \\
0 & \rho_{2} & \cdots & \cdots \\
\cdots & \cdots & \cdots & \cdots \\
\cdots & \cdots & 0 & \rho_{n}
\end{array}\right) .
$$

In the same representation the elements $L_{v}$ we mark as $l_{i j}$. For example, at $\rho_{2}=\cdots=\rho_{n}=0$ calculation of the diagonal elements $M_{i i}$ of the matrix in brackets in (16) gives

$$
M_{11}=-2 \rho_{1} \sum_{i=2}^{n}\left|l_{1 i}\right|^{2}, M_{j j}=2 \rho_{1}\left|l_{1 j}\right|^{2} .
$$

The trace $M_{11}+\cdots+M_{n n}$, as one would expect, is equal to zero. From (52) it is clear that the diagonal elements $\rho_{1}, \rho_{2}, \cdots, \rho_{n}$ as time functions submit to the symmetrical system of the evolutionary equations of the Markov processes. On proved above, the combination

$$
-\sum_{i=1}^{n} \rho_{i}(t) \ln \rho_{i}(t)
$$

monotonically increases. According to the Schur theorem [7] the union of the diagonal elements $\rho_{i}$ is majorized in Hardy, Littlewood and Pais sense by characteristic numbers $\lambda_{i}$ of the same matrix $\rho$. From here follows

$$
\begin{aligned}
S(t) & =-\sum_{i=1}^{n} \lambda_{i}(t) \ln \lambda_{i}(t) \geq-\sum_{i=1}^{n} \rho_{i}(t) \ln \rho_{i}(t) \\
& \geq-\sum_{i=1}^{n} \rho_{i}(0) \ln \rho_{i}(0),
\end{aligned}
$$

i.e.

$$
\begin{aligned}
\rho(t) & =\rho(0)-\gamma t\left[x \psi^{\prime}(x) \psi\left(x_{1}\right)-x \psi^{+}(x) \psi^{\prime}\left(x_{1}\right)+x_{1} \psi^{\prime}\left(x_{1}\right) \psi^{+}(x)-x_{1} \psi\left(x_{1}\right) \psi^{+\prime}(x)\right]+\ldots \\
& =\left[\psi^{+}(x)-\gamma x \psi^{+\prime}(x)\right]\left[\psi\left(x_{1}\right)-\gamma t x \psi^{\prime}\left(x_{1}\right)\right]-\gamma t x \psi^{+}(x) \psi^{\prime}\left(x_{1}\right)+\gamma t x_{1} \psi\left(x_{1}\right) \psi^{+\prime}(x)+O\left(\gamma^{2}\right) .
\end{aligned}
$$

Last two terms in the right member (57) always it is possible to present in the form

$$
\begin{aligned}
& \gamma t\left[\xi x \psi^{+}(x)+\eta \psi^{+\prime}(x)\right]\left[\xi^{*} x \psi(x)+\eta^{*} \psi^{\prime}(x)\right] \\
& -\left[\xi x \psi^{+}(x)-\eta \psi^{+\prime}(x)\right]\left[\xi^{*} x \psi(x)-\eta^{*} \psi^{\prime}(x)\right]
\end{aligned}
$$

with complex coefficients $\xi$ and $\eta$, selected so that the functions

$$
\xi^{+} x \psi(x) \pm \eta^{+} \psi^{\prime}(x)
$$

were crossly orthogonal; an orthogonality of both functions to $\psi(x)$ follows from difference of parity. Then in the right member (57) it is had standard decomposition with one negative coefficient, i.e. obtained a sign-indefinite form, contrary to the instructions in [1]. 
Exclusion makes a case when one of combinations (59) is equal to zero, i.e. at Gaussian shape $\rho\left(x, x_{1}\right)$. Then, apparently, contradictions do not originate and at least in case $H=0$ the functional Equation (55) is reduced to system of the ordinary differential equations. Indeed, let

$$
\rho=c e^{-\alpha x^{2}-\alpha^{+} x_{1}^{2}-2 \beta x x_{1}},
$$

where on the norming condition should be

$$
c=\sqrt{\frac{\alpha+\alpha^{+}+2 \beta}{\pi}} .
$$

For an regularity behavior of the function (60) it is necessary to induct restrictions

$$
\operatorname{Re} \alpha>0, \quad|\beta|<\operatorname{Re} \alpha .
$$

Using (56), we uncover (55):

$$
\frac{\partial c}{\partial t}-c\left(\frac{\mathrm{d} \alpha}{\mathrm{d} t} x^{2}+\frac{\mathrm{d} \alpha^{+}}{\mathrm{d} t} x_{1}^{2}+\frac{\mathrm{d} \beta}{\mathrm{d} t} x x_{1}\right)=-\frac{D}{\hbar^{2}} c\left(x-x_{1}\right)^{2}-2 \gamma c\left[(\beta-\alpha) x+\left(\alpha^{+}-\beta\right) x_{1}\right]\left(x-x_{1}\right) .
$$

Function $c(t)$ of a special role does not play, as is automatically defined by the norming condition. From the common Equation (63) remains

$$
\begin{aligned}
& \frac{\mathrm{d} \alpha}{\mathrm{d} t}=\frac{D}{\hbar^{2}}+2 \gamma(\beta-\alpha), \\
& \frac{\mathrm{d} \beta}{\mathrm{d} t}=-\frac{D}{\hbar^{2}}-\gamma\left(2 \beta-\alpha-\alpha^{+}\right) .
\end{aligned}
$$

The decision of the linear Equation (64) is easy

$$
\begin{aligned}
& \alpha(t)=\varphi-\frac{D}{4 \hbar^{2} \gamma}+i \kappa e^{-2 \gamma t}-g e^{-4 \gamma t}, \\
& \beta(t)=\varphi+\frac{D}{4 \hbar^{2} \gamma}+g e^{-4 \gamma t}, \quad c=\text { const }
\end{aligned}
$$

where $\varphi, g, \kappa$ are the integration constants.

On the other hand, $P_{0}(x)$ should be the solution for (1) without the left part, so after multiplication on $\ln \frac{P_{0}(x)}{P(x)}$ and integration

$$
\iint P_{0}\left(x_{1}\right) \ln \frac{P_{0}(x)}{P(x)} q\left(x_{1}, x\right) \mathrm{d} x \mathrm{~d} x_{1}-\iint P_{0}(x) \ln \frac{P_{0}(x)}{P(x)} q\left(x, x_{1}\right) \mathrm{d} x \mathrm{~d} x_{1}=\iint P_{0}\left(x_{1}\right) \ln \frac{P_{0}(x)}{P_{0}\left(x_{1}\right)} \frac{P\left(x_{1}\right)}{P(x)} q\left(x_{1}, x\right) \mathrm{d} x \mathrm{~d} x_{1}=0 .
$$

Subtraction gives

$$
\begin{gathered}
\frac{\mathrm{d} \widetilde{S}}{\mathrm{~d} t}=\iint\left[P\left(x_{1}\right)-P_{0}\left(x_{1}\right)\right] \ln \frac{P_{0}(x) P\left(x_{1}\right)}{P(x) P_{0}\left(x_{1}\right)} q\left(x_{1}, x\right) \mathrm{d} x \mathrm{~d} x_{1} . \\
\frac{\mathrm{d} S}{\mathrm{~d} t}=\int\left(\frac{2 a-\frac{\mathrm{d} b}{\mathrm{~d} x}}{b}-\frac{1}{f} \frac{\mathrm{d} f}{\mathrm{~d} x}\right)\left[\left(a-\frac{1}{2} \frac{\mathrm{d} b}{\mathrm{~d} x}\right) f-\frac{1}{2} \frac{\mathrm{d} f}{\mathrm{~d} x}\right] \mathrm{d} x=\int \frac{\left[\left(2 a-\frac{\mathrm{d} b}{\mathrm{~d} x}\right) f-b \frac{\mathrm{d} f}{\mathrm{~d} x}\right]^{2} \mathrm{~d} x}{2 b f}>0 .
\end{gathered}
$$

\section{About Paths of Solution of the Schrödinger's Cat Paradox}

The previous notes prepare for thought that a solution of the problem of transition from quantum world in classical world inherently cannot be simple. About it we will underline that in this problem it is impossible to look back. Different versions of "the latent parameters" (in classical sense), still seriously considered in the middle of the XX-th century [19], are now rejected by all development of the quantum mechanics, its interior logical organization and doubtless successes. Now it is necessary to take simply unusual of the microworld which in the general-theoretical sense can be interpreted as considerably greater, in comparison with a macrocosm, an information capacity [20]. In particular, the Bell's ine- qualities [21] hardly deserve their so frequent analysis in up-to-date controversies [22] just because in the developed apparatus of quantum mechanics in them there is no necessity. If the Bell's inequalities nevertheless were carried out, it would mean not simply strangeness, and any full illogicalness of the quantum world that, fortunately, doesn't happen.

The transition from micro- to macro world, figuratively speaking, can be represented by a narrow neck of the bottle, through which one capacious in the information regarding a microcosm of our macrocosm can leak only a very small piece of information. Note also that choice of the one variant among many ones means a decrease in entropy, in contrast to its increase, which is characteristic of the process, incidentally, is also reversible, microscopic interaction with the background of an 
infinite temperature.

Clearly, the equation describing the transition from micro- to macro world must be non-linear one, otherwise, according to Schrödinger, would be possible superposition of the states of live and dead cat. But the search for appropriate nonlinearity randomly or blindly [23] is not productive - an experiment all such attempts quite easily refutes $[24,25]$.

And already now we can tell confidently enough that nonlinear members should have, first, all-round influence, secondly, smooth in space and time, as a result to be almost or not so not found out within the limits of the microcosm. More precisely, the first condition means that there should not be a regular preference which could collect eventually and deform known statistical regularities. In particular, apparently, it is necessary to be careful not to introduce members which would break the law of conservation of energy. Under the smoothness of the action we mean the absence of unmotivated scattering centers and a sufficiently large characteristic time greater than the most common atomic and molecular transformations. The essence of the nonlinear interaction we see a slow non-directional change of the phases and amplitudes of the basis wave functions, for example, characterizing the standard motion of individual molecules in a gas. Stealth aftereffects then follows simply from the fact that these nonlinear effects have "to sink", i.e. disguised among ordinary perturbation (collision of gas molecules, etc.).

Another matter-the states that include the change of macroscopic objects, for example, the "Schrödinger's cat". Conventional thermodynamic fluctuations do not connect them and do not interfere with nonlinear effects. The choice is ultimately one of the macroscopic state of the two, or many, and the resulting decrease in entropy achieved in all probability, only one way: assumptions that nature doesn't suffer superposition of macroscopic states of a certain type, and that among them there is a diffusion, as in the well-known simplified the problem of the "gambler's ruin" until the matter is not reduced to a single, macroscopically admissible states. Sketch of such theory is given in [27]. But with non-linear equations do not get exact performance of the Born postulate: "the probability of a macroscopic event is proportional to the square of the amplitude of the wave function". However, it should be noted: experimental verification of the Born postulate - not out of the circle of those results for which the successful testing of many decimal places is a matter of pride physicists. In fact, exactness of the Born postulate in treatment of the electron diffraction experiments, etc., in general, although satisfactory from the standpoint of practical needs, but in the long term it is necessary to consider possibility of essential infringement of the Born postulate in some special experiments.

The widespread objection that nonlinearity destroys all habitual apparatus of the quantum mechanics, is substantially based on fear before new and unusual which in the twentieth century beginning in the same way prevented to recognize and the general theory of relativity - after all the gravitational field equations on Newton are linear, remarkably convenient and symmetric. On the other hand, are known examples when even sharply nonlinear in itself phenomenon outwardly acts as effect of certain linear forces [28]. Similar things - a harmonious and statistical linearization of control systems [29].

\section{The Cosmic Factors}

For us, it is clear that the source of the diffusion transformation of the wave function should be not in itself (otherwise it would be a regular evolution for no randomness), but something external. After all, even ordinary diffusion of an atom or an electron has its own random factors in the form of aftershocks of the neighboring particles. In problem of the "Schrödinger's cat" should look for this external factor in the macroscopic conditions? In principle, perhaps, but a few plausible, because the macroscopic parameters, in general, varies smoothly and in a regular manner, then the result of the process as even the act of radioactive decay clearly would depend on the macro environment, which is not observed. It remains to assume that it is a factor at all parties for the bodies involved in the experiment. It is likely the effect of cosmic factors. Confirmation is found correlations in geographically separate experiments with macroscopic fluctuations: the correlation as a mutual, and with the astronomical factors [30,31].

\section{Whether the Collapse of Quantum States in the Equilibrium Environment Is Necessary?}

An important conclusion from the foregoing is, we think, very similar tending to statistical equilibrium of manyparticle system on the one hand, and single particle or even a small system surrounded by a stationary background, on the other side. In any case, statistical equilibrium (if it exists) corresponds to the "potential well", to which the system and aims to be simple, monotonous way.

While we did not talk about any collapse or splitting of the wave packets. But there is a need for equilibrium systems (e.g., gases) to consider processes with collapses of the wave functions? For example, in [1], it seems, allowed the existence of such processes with the collapse of a wide range of phenomena closer to equilibrium. In 
contrast to [1], we are inclined to answer this question in the negative if only because it the carefully grounded quantum statistical mechanics [32,33] does not rely on anything like a collapse that would only interfere. To describe the same macroscopic characteristics of the body are, in principle, clearly defined rules, practically independent of the general theoretical views on the nature of the relationship between classical and quantum worlds. In each thermodynamic equilibrium body an entanglement of the quantum states has already reached the maximum extent possible, and continues to move just nowhere. But it is not clear why this should interfere with normal communication with macroscopic bodies as a whole with the classical objects.

You can certainly raise the issue of fluctuations. But the fluctuations in equilibrium systems are not available to our perception. For example, there is no contradiction to say that the fine particles (although the virus) undergoes Brownian motion is completely under the laws of quantum mechanics. But to see this movement, it is necessary to put the object beam, i.e., to create a highly non-equilibrium conditions. Likewise, for example, fluctuations in electrical resistance are a thing in itself, until a sample is not omitted current, and fluctuations in atmospheric density can not be noticed until after it did not get addressed, i.e., again, non-equilibrium radiation, etc. But with creation of non-equilibrium, the problem of fluctuations is transferred to another plane.

\section{The Systems with Large Dispersion}

It must say little about interesting case where the equilibrium state is not reached at least once until the dispersions of the values $q$ and $p$, under a certain typical frequency $\omega$, not to exceed substantially the Heisenberg limit. The formal apparatus of quantum mechanics this did not prevent. For example, for Gaussian density function of the product

$$
\left\langle q^{2}\right\rangle \cdot\left\langle p^{2}\right\rangle=\frac{\hbar^{2}}{4} \frac{|\alpha|^{2}-\beta^{2}}{(\operatorname{Re} \alpha-\beta)^{2}},
$$

bounded below the certain limit $\frac{\hbar^{2}}{4}$, but the rise to infinity occurs $\beta=$ const, $\operatorname{Re} \alpha \rightarrow \beta$. Authors [1,34], facing with like examples seems to be thinking about action of sertain supplementary law, which automatically converts corresponding states in the category of classic ones. However, this can hardly agree. Indeed, in statistical plan the states with high values of dispersion (that practically equivalent growing of the temperature of the background) with good accuracy obey to the classical mechanics laws. But that is not directly related with the issue of choosing a line of the macroscopic development, and does not solve the Schrödinger's cat paradox. The very same simplest (in words) assumptions: "the wave packet splits in achieving of sufficient randomness" is clearly not held, as seen from the inconsistency of the various phenomena of this kind in [9] and from experimental fact of the manifestation of interference to account disguised correlations under, seamingly, the chaotic density function of an atom. In this regard, there are illustrative examples of the photon [35] and plasma [36] echo.

\section{Prospects}

The instances specified above underline that the logic of development of the physical science demands in proximal years of assimilation of the nonlinear quantum mechanics. Such point of view has been stated and justified by us in [27]. There is the mathematical instance of construction of the nonlinear quantum mechanics and considered set of partial cases on description of evolution of the wave function in intermediate zone (the mesoworld) between the microworld and the macroworld. Essential there is an understanding of processes of scattering of the wave packet, and also that the Born's postulate has restricted character. In this connection remains to add the following. Apparently, the important role in processes of a decomposition of the wave packets is played the random character of an energy spectrum on the intermediate stage of the mesoworld. According to instances given in [37], the randomness often originates at interacting of highly exited particles or molecules with environmental small collectives, that is typical for the mesoworld. Dispersion of the wave packets happens, apparently, on clusters of atoms or molecules with characteristic sizes $10^{3}-10^{4} \AA$ Á. From the physical point of view the dispersion on cluster corpuscles is presented by the formula [38]

$$
I(v) \sim v^{D_{S}-6}
$$

where $I$-the intensity of scattered radiation, $D_{S}$ - the fractal dimension of the cluster scattering surface. We pay attention to random character of energy spectrums also because the systems with regular spectrum are studied enough: their quantum properties corresponding to the usual theory are often prolonged in the macrocosm in the form of various macroscopic quantum appearances. Therefore the greatest chances to find out the quantum nonlinearity are available there where for an interference of wave packages earlier didn't search.

The reference to quasi-stationary states in [1] also serves, inevitably at first, to simplification of an essence of the matter. Most likely, at more exact analysis it is necessary to connect external world influences with tran- 
sitions between different conditions. Otherwise is not absolutely clear, as it is possible to register quantum jumps [39] in a state of separate or several atoms. In describing the atom simply the density function without taking into account the dynamics of interaction with the background would have been a simple stationary, does not give grounds for splitting.

Let's address now to effect of Sokolov. In Sokolov's experiments [40] - here we enter on shaky groundsconfuses independence of the effect in an aspect of "the Demon field" from a beam intensity of atoms so the nonlinearity of the Schrodinger equation vanishes. We offer several other interpretation of the Sokolov's experiment: apparently, the surface of the sample of metal by which microparticles (atoms of hydrogen) flew by, in these experience was in the special, hardened state when in surface layer a quantum superposition of collective states gradually are destroyed by interacting with separately flying atoms, which execute role of a certain catalytic process. Then each atom of hydrogen calls the effect, and effects are simply summed up. If our assumption of the reason of the effect is truly, has to be observed a gradual "aging", or effect exhaustion at the long-term use of installation with the same piece of metal (from the published reports of Sokolov's effect not clearly, whether there is such process of an aging actually). By the way, finiteness of a time of "viability" of equipment detecting cosmic impacts (not quite us comprehensible) it is underlined in [41].

With under discussion by complex of the problems, probably, are bound events of the enigmatic delay to registrations of the effect in absolutely different experiments. Here pertains emission of Rydberg's atoms in resonator [42], and also the quantum effect of Zeno [43] and registration of wide atmospheric showers with energy $\sim 10^{16}$ эB [44]. Here should be adding on and established experiments with macroscopic fluctuations in the registration of radioactive decay, etc. What has shown, in particular, the following debate in UFN, results on explanation of macroscopic fluctuations do not pack nor in what rules of the statistics if consider that registration goes in mode of the realtime - thence need to postulate the delay of the order of the second. At last, though here it is not visible to connection with quanta yet, there is a problem of rare "delayed echo" in a radio communication [45], not explainable, on condition of observation, reflexion from something. Thus, the impression is made that sometimes the nonlinear quantum process detain on intervals of the order of second an information exit in our usual world. Perhaps these facts are actually more, but they are hidden in small editions available or not published under the pretexts of strangeness.

In summary, unlike [23], we will underline objective character of a problem of the transition a microcosmsmacrocosm. Just research in recent decades clearly demonstrate the reality of the processes occurring in the intermediate scale, in contrast to the era of birth of quantum mechanics, where the transition from the processes of the microworld seemed something of a jump across the gap. Now this gap is gradually filled. Continue to close our eyes to mezoworld as important as physically real band - then advance to build barriers to learning. In experiments with microobjects we deal with facts as real as the phenomena that surround us. A suitable analogy might be a statistical statement: malaria incidence is higher in wetlands. Very poor would have been in our time, one epidemiologist, who announced to the swamp "of the primary accident" and thus would have missed the most important factor-the mosquito!

\section{Conclusions}

Some characteristics of the Markov chains (processes) are specified and the important property of monotonicity of a probability is formulated. It is entered notion to relative entropy and is found monotonous nature its behaviour in classical case. For study of irreversibility phenomena in quantum problems by new methods is received the Lindblad's equation and its physical essence is explained. Deep analogy between the classical Markov processes and development described by the Lindblad's equation is conducted. Using method of comparison of the Lindblad's equation with the linear Langevin equation we receive a system of differential equations, which are more general, than the Caldeira-Leggett equation. We consider quantum systems without inverse influence on a surrounding background with high temperature. Quantum diffusion of a single particle is considered and possible ways of the permission of the Schrödinger's cat paradox and the role of an external world for the phenomena with quantum irreversibility are discussed. In spite of previous opinion we conclude that in the equilibrium environment is not necessary to postulate the processes with collapses of wave functions. We draw attention to the fact that the Heisenberg's uncertainty relation does not always mean the restriction is usually the product of the average values of commuting variables. It is discussed row new experiments, in accordance with under discussion problem of the quantum nonlinearity. We underline that the logic of development of the physical science demands in proximal years of assimilation of the nonlinear quantum mechanics.

\section{References}

[1] M. B. Mensky, "Dissipation and Decoherence of Quan- 
tum Systems," UFN, Vol. 173, 2003, pp. 1199. doi:10.3367/UFNr.0173.200311d.1199

[2] S. Karlin, "Mathematical Methods and Theory in Games, Programming, and Economics," Dover Publications, 1992.

[3] C. KayLay, "Uniform Chains of the Markov," Mir, Moscow, 1964 (in Russian).

[4] M. S. Bartlett, "Introduction to Theory of the Probability Processes," Inostrannaya literatura, Moscow, 1958, (in Russian).

[5] M. Klein, "Entropy and the Ehrenfest urn Model," Physica, Vol. 22, No. 6-12, 1956, pp. 569-575.

[6] M. Loeve, "Probability Theory," 4th Edition, Springer, 1977.

[7] A. Marshal and I. Olkin, "Inequality," Academic Press, New York, 1979.

[8] S. Chandrasekhar, "Principles of Stellar Dynamics," Dover, New York, 1942.

[9] B. B. Kadomcev, "Dynamics and Information," YFN, Moscow, 1999 (in Russian).

[10] G. B. Lesovik, "Reservoir as Source of Quantum Mechanical Probability," Letter in JETP, Vol. 74, No. 9, 2001, pp. 528.

[11] G. Lindblad, "On the Generators of Quantum Dynamical Semi Groups," Communications in Mathematical Physics, Vol. 48, No. 2, 1976, pp.119-130. doi:10.1007/BF01608499

[12] R. Balesku, "Equilibrium and Nonequilibrium Statistical Mechanics," Wiley \& Sons, New York, 1975.

[13] E. M. Lifshic and L. P. Pitaevskiy, "Fizicheskaya Kinetika," (Physical Kinetics), Nauka, Moscow, 1979, (in Russian).

[14] J. V. Pulé, "The Bloch Equations," Communications in Mathematical Physics, Vol. 38, No. 3, 1974, pp. 241-256. doi:10.1007/BF01651544

[15] H. Bateman and A. Erdelyi, "Higher Transcendental Functions," Mc Graw-Hill Book Company, New York, 1953.

[16] P. K. Suetin, "Classical Orthogonal Polynomials," Nauka, Moscow, 1979, (in Russian).

[17] A. O. Caldeira and A. J. Leggett, "Path Integral Approach to Quantum Brownian Motion," Physica A, Vol. 121, No. 3, September 1983, pp. 587-616. doi:10.1016/0378-4371(83)90013-4

[18] A. O. Caldeira and A. J. Leggett, "Influence of Damping on Quantum Interference: An Ex Aptly Soluble Model," Physical Review A, Vol. 31, No. 2, 1985, pp. 1059-1066. doi:10.1103/PhysRevA.31.1059

[19] "Philosophical Questions of Modern Physics," M.: ADVO AN USSR, 1952 (in Russian).

[20] I. A. Akchurin, "Theory of the Elementary Particles and Theory to Information-Philosophical Problems of Physics of Elementary Particles," Nauka, Moscow, 1964, (in Russian).

[21] A. A. Grib, “The Bell's Inequality and Experimental
Check of Quantum Correlation on Macroscopic Distances," UFN, Vol. 142, 1984, pp. 619-634. doi:10.3367/UFNr.0142.198404d.0619

[22] M. A. Popov, "In Protection of the Quantum Idealism," UFN, Vol. 173, 2003, pp. 1382-1384. doi:10.3367/UFNr.0173.200312j.1382

[23] I. Bialynicki-Birula and J. Mycielski, "Nonlinear Wave Mechanics," Annals of Physics, Vol. 100, No. 1-2, 1976, pp. 62. doi:10.1016/0003-4916(76)90057-9

[24] C. G. Shull, D. K. Atwood, J. Arthur and M. Horne, "Search for a Nonlinear Variant of the Schrödinger Equation by Neutron Interferometry," Physical Review Letters, Vol. 44, No. 12, 1980, pp. 765-768. doi:10.1103/PhysRevLett.44.765

[25] R. Gähler, A. Y. Klei and A. Zeilinger, "Neutron Optical Tests of Nonlinear Wave Mechanics," Physical Review A, Vol. 23, No. 4, 1981, pp. 1611-1617.

[26] B. P. Kondratyev and V. A. Antonov, "The Solution of the Schrödinger's Cat Paradox. Attempt of Development of Nonlinear Quantum Mechanics," Izhevsk, Udmurt State University, 1994 (in Russian).

[27] Y.A. Panovko and I. I. Gubanova, "Stability and Oscillations of the Springy Systems," Nauka, Moscow, 1979, (in Russian).

[28] V. S. Pugachev, "Bases of the Autocontrol," Fizmatgiz, Moscow, 1963, (in Russian).

[29] S. E. Shnoll, V. A. Kolombet, E. V. Pozharskiy, T. A. Zinchenko, I. M. Zvereva and A. A. Konradov, "About Realization of Discrete Conditions during Fluctuations in Macroscopical Processes," UFN, Vol. 10, 1998, pp. 1129-1140. doi:10.3367/UFNr.0168.199810e.1129

[30] S. E. Shnoll, "Macroscopical Fluctuations of the Form Discrete Distributions Cosmophysical Reasons," Biophysics, Vol. 46, No. 5, 2001, pp. 733-741.

[31] B. B. Kadomcev, "Irreversible in Quantum Mechanics," UFN, Vol. 173, 2003, pp. 1221-1240. doi:10.3367/UFNr.0173.200311e.1221

[32] L. P. Kadanoff and G. Baym, "Quantum Statistical Mechanics," Mir, Moscow, 1964, (in Russian).

[33] I. D. Abella, N. A. Kurnit and S. R. Hartmann, "Photon Ehoes," Physical Review, Vol. 141, No. 1, 1966, pp. 391406. doi:10.1103/PhysRev.141.391

[34] A. Y. Hinchin, "Mathematical Basis Statistical Mechanics," Gostehizdat, Moscow, 1947, (in Russian).

[35] V. N. Pavlenko, "The Eho Phenomena in Plasma," UFN, Vol. 141, No. 3, 1983, pp. 393-429. doi:10.3367/UFNr.0141.198311a.0393

[36] M. Zaslavsky, "Statistics of the Energy Spectrum," UFN, Vol. 129, 1979, p. 211.

[37] M Schroeder, "Fractals, Chaos, Power Laws: Minutes from an Infinite," Paradise, New York, 1991.

[38] K. N. Drabovich, "Captivated Atomic Particles in Action," UFN, Vol. 158, No. 3, 1989, pp. 499-510. doi:10.3367/UFNr.0158.198907e.0499

[39] Y. L. Sokolov "Interference Method of the Measurement Parameters Atomic Conditions," UFN, Vol. 169, No. 5, 
1999, pp. 559-583. doi:10.3367/UFNr.0169.199905f.0559

[40] A. M. Mishin, "Problems of Research of the Universe," Publishing House of St. Petersburg State University, St. Petersburg, 2001, (in Russian).

[41] I. M. Beterov and P. B. Lerner, "Spontaneous and Forced Radiation of Rudberg's Atom in Resonator," UFN, Vol. 159, No. 4, 1989, pp. 665-711. doi:10.3367/UFNr.0159.198912c.0665

[42] W. H. Itano, D. J. Heinzen, J. J. Bottlinger and D. J.
Wineland, "Quantum Zeno Effect," Physical Review A, Vol. 41, No. 5, 1990, pp. 2295-2300.

doi:10.1103/PhysRevA.41.2295

[43] B. Zhdanov and A. P. Chubenko, Materials of the Seminar Devoted to the 80 Anniversary from the Date of Birth of M.I. Podgoretsky, Dubna, 2000, (in Russian), p. 32.

[44] L. M. Gindilis, "Model of the Contact, Rather Then Proof of the Probe," Earth and Universe, No. 2, 1976, (in Russian), p. 78 . 